\title{
Congenital Bilateral Choanal Atresia with Duodenal Atresia and Lagophthalmos
}

\author{
${ }^{1}$ Sanjeev Gupta, ${ }^{2}$ Suryakanta Pradhan
}

\begin{abstract}
Bilateral choanal atresia is an uncommon congenital malformation and its association with duodenal atresia and lagophthalmos is very rare. It is a life-threatening condition where immediate intervention is always needed. A systemic examination is required to find its association with other anomalies. Though different approaches for its treatment are described endoscopic transnasal approach is the most acceptable less traumatic approach till date. We are having a case report of a premature baby with choanal and duodenal atresia with lagophthalmos and was managed successfully with endoscopic transnasal approach.
\end{abstract}

Keywords: Choanal atresia, Duodenal atresia, Endoscopic transnasal approach, Lagophthalmos.

How to cite this article: GuptaS, Pradhan S. Congenital Bilateral Choanal Atresia with Duodenal Atresia and Lagophthalmos. Int J Otorhinolaryngol Clin 2014;6(3):137-139.

Source of support: Nil

Conflict of interest: None

\section{INTRODUCTION}

Congenital choanal atresia is a rare anomaly. Its incidence is one in 7000 live births. ${ }^{1}$ It may be unilateral or bilateral. It seems to occur more commonly in females than males and is frequently unilateral and right-sided than bilateral. The risk is slightly more in twins. Chromosomal anomalies are found in some of the babies. ${ }^{2}$ It may be bony, cartilaginous or mixed. Current theories of choanal atresia embryogenesis cite either failed oronasal membrane rupture or abnormal migration of neural crest cells into the nasal vault. ${ }^{3,4}$ Lee et al in their study found $\mathrm{T}_{4}$ level is low in most of the babies having choanal atresia which may be a risk factor for the anomaly. ${ }^{5}$ Most of the time choanal atresia is an isolated entity but it may be associated with charge syndrome (coloboma, heart defect, atresia choanae, retardation of growth, genital anomalies and ear anomalies). Bilateral choanal atresia in newborn causes cyclical cyanosis and asphyxia which

${ }^{1}$ Senior Consultant, ${ }^{2}$ Registrar

1,2Department of ENT and Head Neck Surgery, Apollo Hospitals Bhubaneswar, Odisha, India

Corresponding Author: Suryakanta Pradhan, Registrar, Department of ENT and Head Neck Surgery, Apollo Hospitals Bhubaneswar, Odisha, India, Phone: 8093060163 , e-mail: drsuryakanta83 @gmail.com gets relieved by crying as the mouth opens and when the crying stops the obstruction repeats. The asphyxia may lead to cardiopulmonary arrest and death if not managed appropriately. After birth if choanal atresia is suspected complete physical examination is done to rule out any other syndromic association. A small sized nasogastric tube or catheter is passed through the nostrils to choana to check the patency but CT-scan PNS gives the most accurate diagnosis. Other investigations in case of choanal atresia should include echocardiography, ultrasonography of the abdomen, ophthalmological evaluation and otorhinolaryngology evaluation.

\section{CASE REPORT}

We are presenting a case of a preterm baby boy of birth weight $2.37 \mathrm{~kg}$ born by emergency LSCS to 5 th gravida mother. Antenatally diagnosed as a case of duodenal atresia as she was having polyhydramnios and double bubble sign on USG. After birth he immediately developed cyclical spells of cyanosis and asphyxia which was getting relieved by crying. Mouth gag was placed but it was insufficient to maintain the saturation. Patient was intubated immediately and ventilated. We have tried to put nagogastric feeding tube but there was some obstruction in the nasal cavity. He was also having lagophthalmos of the right eye. Rest physical examinations were normal without any cardiac or genital abnormality.

He was investigated with contrast study and CT scan PNS. Screening CT scan and X-ray chest and abdomen confirmed the diagnosis of duodenal atresia (Fig. 1). Serial axial sections of paranasal sinuses were performed with coronal and sagittal reconstructions. All the sinuses were normal. There was an abnormal thin soft tissue membrane noted on either side of posterior nasal cavity. Posterior nasal cavity measures $<3 \mathrm{~mm}$ in its maximum thickness. Choanal atresia of membranous variety was confirmed (Figs 2 to 4 ).

Bilateral recanalization for choanal atresia and exploratory laparotomy were planned. Supportive treatment including oxygen, intravenous fluids, and prophylactic antibiotics were started. After improvement of general condition baby was taken for surgery. Nasal endoscopy was done and bilateral choanal atresia visualized. It was recanalized by the thermal probes. It was dilated and 


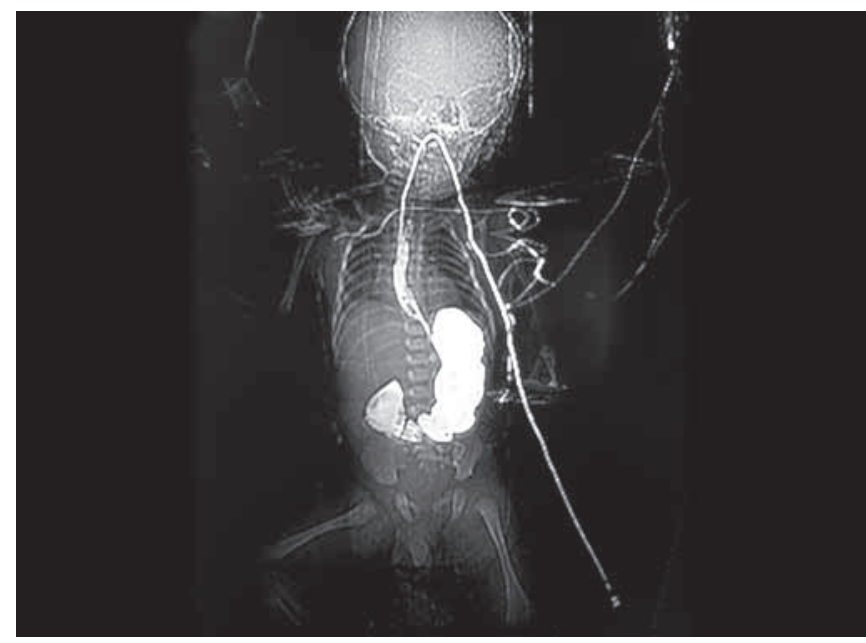

Fig. 1: Screening CT scan showing duodenal atresia

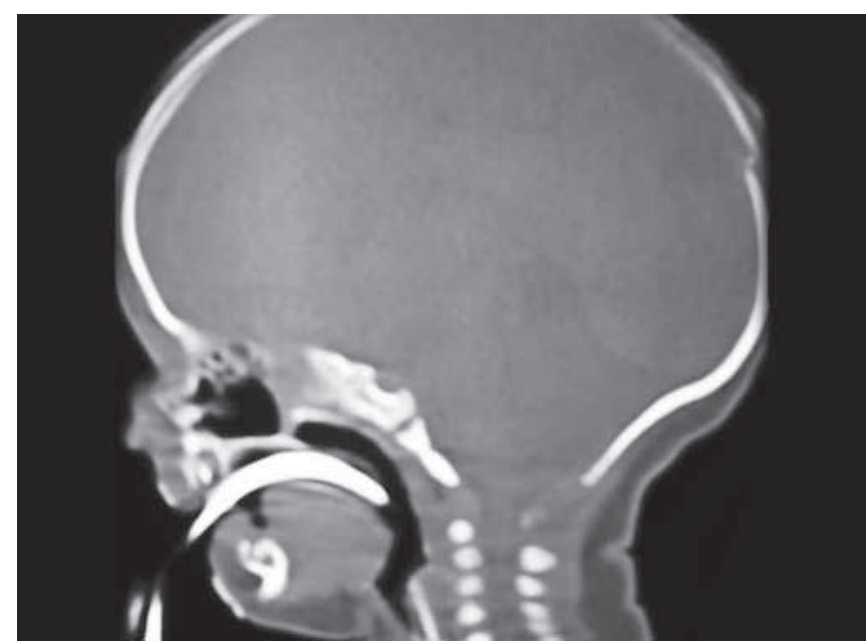

Fig. 3: Computed tomography scan sagittal view showing left nasal cavities with choanal atresia

no. 4 endotracheal tube stents put in both the nostrils and stitched (Fig. 5). Grossly dilated stomach with blindly ending duodenum was found on exploratory laparotomy. Duodeno duodenostomy performed after checking distal patency. Baby was ventilated for about 24 hours and then extubated. He was discharged with stents in place and followed up in OPD. The stents were removed after 1 month. There was small granulation in the choana which was removed. The choana was patent after 6 months of follow-up.

\section{DISCUSSION}

Choanal atresia is a rare condition in which there is a bilateral or unilateral partition at the level of junction of nose and nasopharynx. Unilateral atresia is more common and may present at a later age with nasal blockage or discharge. But the bilateral atresia causes complete nasal obstruction, immediate respiratory distress and even potential death due to asphyxia as

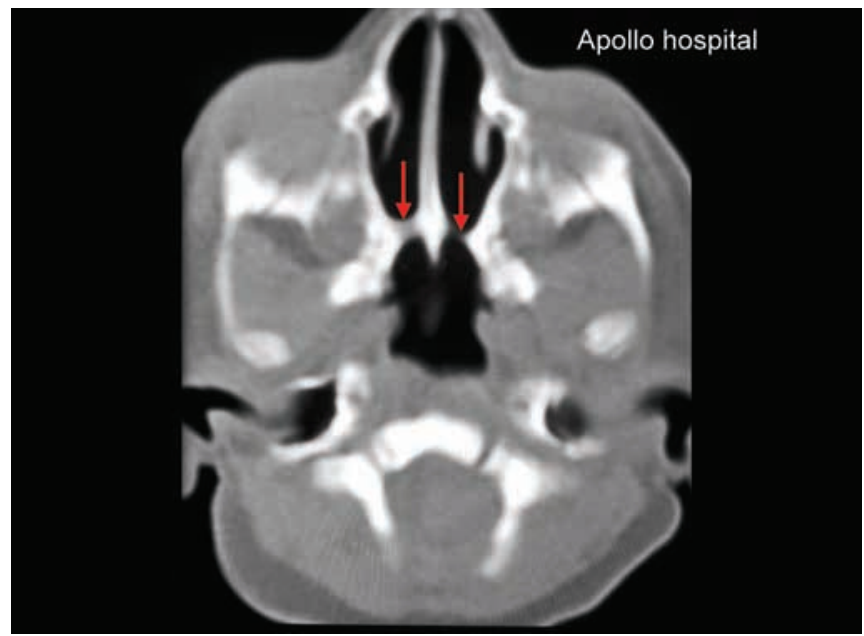

Fig. 2: Computed tomography scan axial view showing both the nasal cavities with choanal atresia

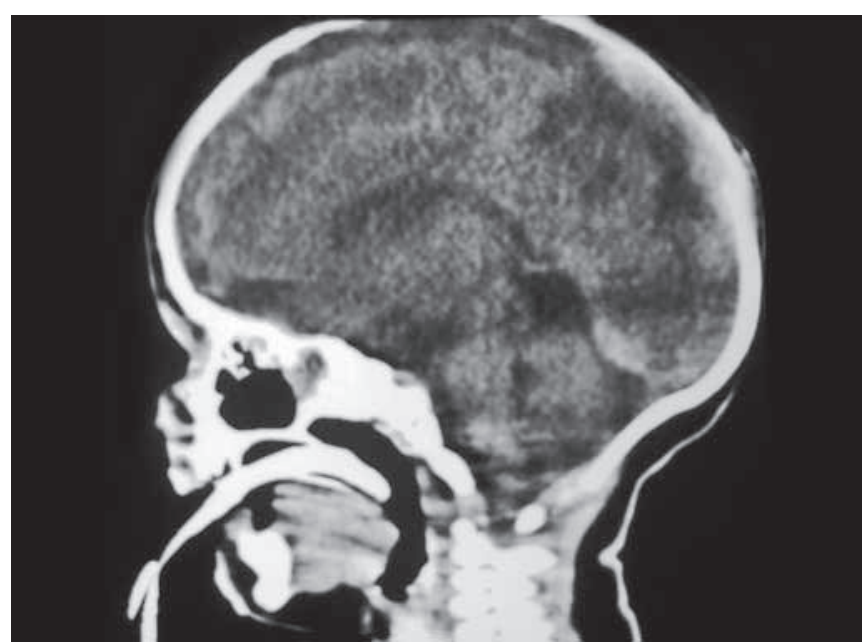

Fig. 4: Computed tomography scan sagittal view showing right nasal cavities with choanal atresia

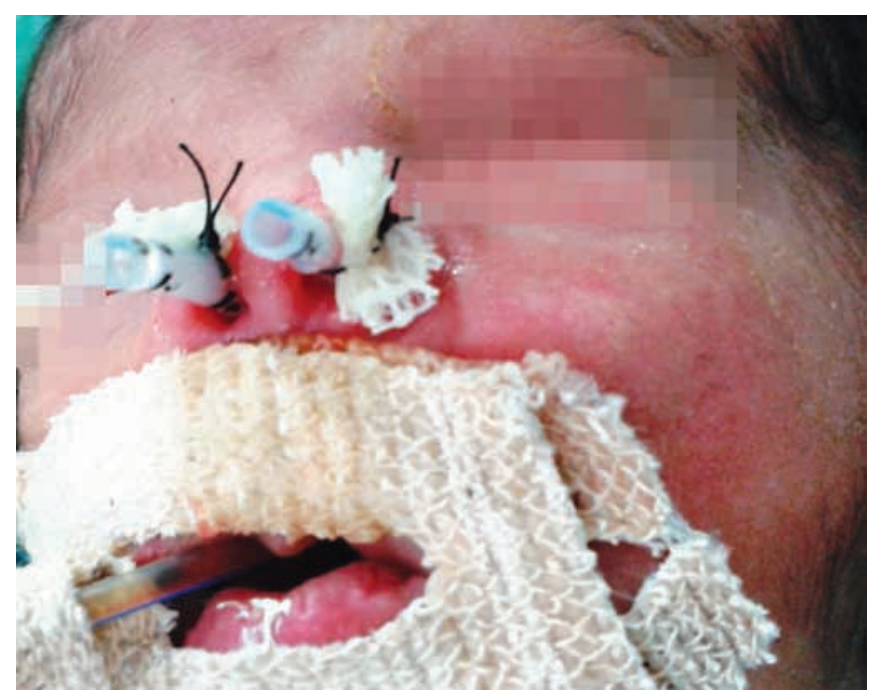

Fig. 5: Postoperative photograph showing nasal stents in place

the newborns are obligate nose breathers. The baby typically presents with cyclical cyanosis, i.e. blue spells relieved by crying. When the baby closes mouth or 
sleeps the saturation decreases and distress increases. Initially, it can be managed by a properly placed mouth gag. But sometimes the respiratory distress is very severe and the baby needs to be intubated. It was first described by Johann Roderer in 1755, male to female ratio is (1:2) and unilateral atresia is more common than bilateral. ${ }^{6}$ The clinical evaluation of a choanal atresia baby should include the examination of cardiovascular system, genitourinary system along with ear and eye. The differential diagnosis should include deviated nasal septum, turbinate hypertrophy, encephalocele, tumors, pyriformaperture stenosis, dermoid or pharyngeal bursa. Choanal atresia is suspected in babies with typical features and inability to pass a suction catheter through nose. The diagnosis is confirmed by CT scan of nose and paranasal sinuses or by rigid or flexible endoscopy. Many patients have association with other diseases as in charge syndrome. But in our case it was associated with duodenal atresia and lagophthalmos which was not described in the literature.

The treatment of unilateral choanal atresia is usually an elective surgery, whereas of bilateral is an emergency one. First airway is secured then the surgery is planned. Different approaches for correction were described but still it is controversial. No technique has been accepted universally till date. The ideal procedure of recanalization should restore the airway without damaging adjacent structures. It can be transpalatine, transnasal, combined transpalatine and transnasal or transseptal. Both transpalatine and transnasal approaches have similar outcomes. ${ }^{7}$

Transpalatine recanalization gives good access and has a good success rate but the intraoperative and postoperative complications are more. Endoscopic transnasal repair has gained popularity in recent times and its results are better with less recovery period in comparison to classical transnasal repair. Use of powered instruments like microdebrider can provide better results. ${ }^{8}$ The success rate for unilateral recanalization is better in comparison to bilateral atresia. Antineoplastic agent mitomycin-C can be used to prevent granulation tissue and fibrosis after the stent removal and also the need for revision surgery. ${ }^{9}$ Newman et al analyzed different factors affecting 15 years surgical outcomes of choanal atresia repair. They found that their revision rate after initial endoscopic repair of choanal atresia was low and was unaffected by adjuvant mitomycin-C therapy or stenting. ${ }^{10}$ Carbon dioxide and KTP lasers can be tried for recanalization. The time of hospitalization and operation is very short with the use of laser. Balloon dilation can be used in case of membranous atresia which is an atraumatic safe OPD procedure.

\section{REFERENCES}

1. Scott-Brown's Otolaryngology, Head and Neck Surgery. 7th ed; vol 1: 1070.

2. Leclerc JE, Fearon B. Choanal atresia and associated anomalies. Int J Pediatr Otorhinolaryngol 1987;13(3):265-272.

3. Heneger AS, Strom M. Choanal atresia. A new embryonic theory and its influence on surgical management. Laryngoscope 2009;92(8):913-921.

4. Dunham ME, Miller RP. Bilateral choanal atresia associated with malformation of the anterior skull base, embryogenesis and clinical considerations. Ann Otol Rhinol Laryngol 1992; 101(11):916-919.

5. Lee LJ, Canfield MA, Hashmi SS, Moffitt KB, Marengo L, Agopian AJ, et al. Association between thyroxine levels at birth and choanal atresia or stenosis among infants in Texas, 2004-2007. Birth Defects Res A Clin Mol Teratol 2012; 94(11): 951-954.

6. Leclerc JE, Fearon B. Choanal atresia and associated anomalies. Int J Pediatr. Otorhinolaryngol 1987;13(3):265-272.

7. Triglia JM, et al. Choanal atresia: therapeutic management and results in a series of 58 children. Revue de laryngologieOtologie-Rhinologie 2003;124:139-143.

8. Van Den Abbeele T, et al. Transnasal endoscopic treatment of choanal atresia without prolonged stenting. Archives of Otolaryngology—Head and Neck Surgery 2002;128:936-940.

9. Prasad M, Ward RF, April MM, Bent JP, Froehlich P. Topical mitomycin as an adjunct to choanal atresia repair. Arch Otolaryngol Head Neck Surg 2002;128(4):398-400.

10. Newman JR, Harmon P, Shirley WP, Hill JS, Woolley AL, Wiatrak BJ. Operative management of choanal atresia: a 15-year experience. JAMA Otolaryngol Head Neck Surg 2013;139(1):71-75. 